\title{
Synovial fluid pyrophosphate and nucleoside triphosphate pyrophosphatase: comparison between normal and diseased and between inflamed and non-inflamed joints
}

\author{
Martin Pattrick, Edith Hamilton, June Hornby, Michael Doherty
}

\begin{abstract}
Deposition of intra-articular calcium pyrophosphate is associated with both aging and arthropathy; increased concentrations of free pyrophosphate (PPi) may contribute to such deposition. Free pyrophosphate and nucleoside triphosphate pyrophosphatase (NTPase) were estimated in synovial fluids from 50 subjects with normal knees and from 44 patients with rheumatoid arthritis, 61 with pyrophosphate arthropathy, and 59 with osteoarthritis. For arthropathic knees clinically assessed inflammation was classified as active or inactive using a summated score of six clinical features.

The order of PPi ( $\mu \mathrm{mol} / \mathrm{l})$ and NTPase ( $\mu \mathrm{mol}$ PPi/30 min/mg protein) was pyrophosphate arthropathy $>$ osteoarthritis $>$ rheumatoid arthritis (median PPi, NTPase respectively: for pyrophosphate arthropathy 15.9, 0.45 ; for osteoarthritis $9.3,0.25$; for rheumatoid arthritis $4 \cdot 4,0 \cdot 18$ ), with significant differences between all groups. In pyrophosphate arthropathy both PPi ( $\mu \mathrm{mol} / \mathrm{l})$ and NTPase ( $\mu \mathrm{mol} \mathrm{PPi} / 30 \mathrm{~min} / \mathrm{mg}$ protein) were higher than normal $(15 \cdot 9,0.45 v 8 \cdot 6,0.2$ respectively), but findings in osteoarthritis did not differ from normal. The inflammatory state of the knee had a distinct but variable effect on synovial fluid findings in rheumatoid arthritis and pyrophosphate arthropathy, but not in osteoarthritis. There was no correlation of either PPi or NTPase with age, or between PPi and NTPase in any group.

This study provides in vivo data for synovial fluid PPi and NTPase. It suggests that factors other than PPi need to be considered in a study of crystal associated arthropathy. Clinical inflammation, as well as diagnosis, is important in synovial fluid studies.
\end{abstract}

Although intra-articular deposition of crystalline calcium pyrophosphate dihydrate (CPPD) most commonly occurs as an identical, age related phenomenon, ${ }^{1}$ such deposition has been associated with 'pyrophosphate arthropathy', a subset of osteoarthritis characterised by typical clinical and radiographic features. ${ }^{3}$ The relation between crystals, inflammation, and arthropathy, however, remains uncertain. ${ }^{1-3}$ The two principal hypotheses suggest that CPPD crystals either are $(a)$ primary pathogenic particles or $(b)$ are formed as a secondary event to joint insult, reflecting processes that accompany the articular response that is recognised as 'osteoarthritis'. 1
Many factors may affect crystal deposition and possibly, intra-articular inorganic pyrophosphate (PPi) concentration is important. Previous studies have estimated PPi in various arthropathies and reported raised concentrations in osteoarthritis synovial fluid. A possible source of excess PPi is through hydrolysis of nucleoside triphosphates spilled by metabolically active, dividing or damaged cells. This might be facilitated by activity of the chondrocyte ectoenzyme nucleoside triphosphate pyrophosphatase (NTPase), which is found 'free' in synovial fluid from osteoarthritic joints. ${ }^{4-16}$ Estimation of PPi and NTPase in biological systems is technically difficult, however ${ }^{16-21}$; various methods have been used, with conflicting results. ${ }^{22-27}$ Furthermore, sample numbers in most studies have been limited, and there is a paucity of data for normal joints. 5

We used a modification of the sensitive and specific assay for PPi developed by McGuire et $a l^{6}$ to estimate $\mathrm{PPi}$ and NTPase in knee synovial fluid from well defined patient groups and from normal controls. For abnormal knees the degree of clinical inflammation at the time of aspiration was assessed by a summated score using six variables ${ }^{28}$; comparison between inflamed and non-inflamed knees, as well as between diagnostic categories, was therefore made.

Subjects and methods

Approval for aspiration of normal knees (by means of the medial infrapatellar approach) was obtained from the local ethical committee.

NORMAL SUBJECTS AND PATIENTS

Normal volunteers ( 29 female, 21 male; median age 44 , interquartile range $26-63$ years) gave informed consent and had no symptoms or clinical signs of knee disease; radiographs were obtained in those aged $>50$ years to exclude occult osteoarthritis or other abnormality. All patients with rheumatoid arthritis fulfilled American Rheumatism Association criteria for classic or definite disease ( 29 female, 15 male; median age 63, interquartile range 54-69 years). Patients with knee osteoarthritis ( 29 female, 30 male; median age 70, interquartile range 63-75 years) had symptomatic gonarthrosis with weightbearing knee radiographs showing cartilage loss plus subchondral sclerosis, osteophyte, or both; none had radiographic chondrocalcinosis, synovial fluid CPPD crystals, or evidence of other primary joint disease. Patients

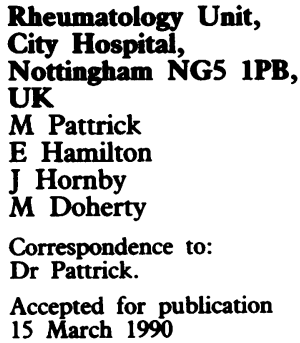


with chronic pyrophosphate arthropathy (40 female, 21 male; median age 77, interquartile range 72-83 years) had persistent ( $>3$ months) symptomatic gonarthrosis with synovial fluid CPPD crystals and radiographic features of osteoarthritis (with or without chondrocalcinosis).

\section{ASSESSMENT OF CLINICAL INFLAMMATION}

For patients with rheumatoid arthritis, osteoarthritis, and pyrophosphate arthropathy each knee was designated as either 'active' or 'inactive' according to clinically assessed inflammation, using a summated score $(0-6)$ of six features: duration of early morning and inactivity stiffness, warmth, joint line tenderness, effusion, and synovial thickening. ${ }^{28}$ Scores of 4-6 were regarded as active, $0-2$ as inactive; knees with intermediate scores were not included in this study.

\section{SAMPLE HANDLING}

All fluids were taken into sterile plastic containers and processed as soon as possible (within two hours). Crystal identification was by characteristic morphology and birefringence using compensated polarised light microscopy of unspun synovial fluid. Samples were centrifuged at $2500 \mathrm{~g}$ for 15 minutes to remove cells and crystals; supernatants were stored at $-20^{\circ} \mathrm{C}$ and assayed within three months.

\section{ASSAY OF INORGANIC PYROPHOSPHATE}

Inorganic pyrophosphate was estimated by a modification of the radiometric assay developed by McGuire et al. ${ }^{16}$ Briefly, the sample PPi was allowed to react with added tritiated uridine diphosphogluconate, and the product, glucose-1phosphate, was converted to a labelled, stable, recoverable product-tritiated 6-phosphogluconate (each sample assayed in duplicate ${ }^{29-32}$ ).

The PPi assay mixture contained $57 \mathrm{mM}$ TRIS acetate; $5.2 \mathrm{mM}$ magnesium acetate; $4 \mu \mathrm{M}$ nicotinamide-adenine dinucleotide phosphate; 18.6 $\mu \mathrm{M}$ glucose-1,6-diphosphate; $7.5 \mu \mathrm{M}$ uridine diphosphogluconate; $0.4 \mathrm{U} / 1$ glucose-6-phosphate dehydrogenase; $0.2 \mathrm{U} / 1$ phosphoglucomutase; $0.136 \mathrm{U} / 1$ uridine diphosphoglucose pyrophosphorylase; and tritiated uridine diphosphogluconate (specific activity $110-370 \mathrm{GBq} / \mathrm{mmol}$ ). A $100 \mu \mathrm{l}$ aliquot of this mixture was incubated with $40 \mu \mathrm{l}$ of either sample or standard PPi $(0.05-0.8 \mu \mathrm{mol} / \mathrm{l})$.

The incubation reaction was performed in triplicate for 75 minutes at $37^{\circ} \mathrm{C}$, then terminated by cooling on ice. A $250 \mu \mathrm{l}$ aliquot of activated charcoal solution (deionised water) was added to separate any unreacted substrate from labelled 6-phosphogluconate. After mixing, samples were centrifuged for 15 minutes at $2500 \mathrm{~g}$ at $4^{\circ} \mathrm{C}$. Supernatant $(200 \mu \mathrm{l})$ was counted for radioactivity in scintillation fluid.

ASSAY OF NTPase ACTIVITY

NTPase activity is expressed as $\mu \mathrm{mol}$ PPi (per mg total protein) generated over 30 minutes in the presence of added ATP.

Aliquots $(200 \mu \mathrm{l})$ of synovial fluid were incubated at $37^{\circ} \mathrm{C}$ in the presence of $120 \mu \mathrm{M}$ ATP and tracer ${ }^{32} \mathrm{P}$ PPI to determine any breakdown of PPi during subsequent incubation and extraction procedures.

The incubation mixture contained $5.4 \mathrm{mM}$ $\mathrm{KCl} ; 0.8 \mathrm{mM} \mathrm{MgSO}$; $1.8 \mathrm{mM} \mathrm{CaCl}_{2} ; 0.118$ $\mathrm{mM} \mathrm{NaCl} ; 1 \mathrm{mM} \mathrm{NaH}{ }_{2} \mathrm{PO}_{4} ; 5.56 \mathrm{mM}$ glucose; and $20 \mathrm{mM}$ HEPES $(N$-2-hydroxyethylpiperazine- $N^{\prime}$-2-ethanesulphonic acid). The mixture was held for 30 minutes in a shaking water bath and then incubation was terminated by cooling on ice and by addition of $0.1 \mathrm{ml}$ trichloroacetic acid $(50 \% \mathrm{w} / \mathrm{v})$. After centrifugation at $2500 \mathrm{~g}$ for 15 minutes at $4^{\circ} \mathrm{C}$ trichloroacetic acid was removed by the addition of one volume of tri-noctylamine dissolved in three volumes of $1,1,2$ trichlorofluoroethane. This mixture draws the trichloroacetic acid into the lower organic layer. The resulting $500 \mu \mathrm{l}$ aqueous extract was neutralised by addition of $100 \mu \mathrm{l} 0 \cdot 1 \mathrm{M}$ TRIS acetate $/ 8 \mathrm{mM}$ magnesium acetate.

To measure any hydrolysis of PPi to orthophosphate during incubation and extraction the following recovery procedure was carried out on each sample. Orthophosphate was removed by mixing $100 \mu \mathrm{l}$ of each sample with an equal volume of ammonium molybdate $(5 \% \mathrm{w} / \mathrm{v})$ in $2.7 \mathrm{M} \mathrm{HCl}$. The resulting phosphomolybdate complex was recovered by extraction into isobutanol/light petroleum $(4: 1 \mathrm{v} / \mathrm{v})$. The remaining ${ }^{32} \mathrm{P} \mathrm{PPi}$ was then counted in the aqueous phase in $0.1 \mathrm{M} \mathrm{HCl}$. These reactions were performed in duplicate. Total synovial fluid protein was estimated by the Biuret method.

\section{ANALYSIS}

Differences between continuously variable data were tested by the Wilcoxon rank sum test, and correlation by Spearman's method.

\section{Results}

Table 1 shows the numbers of synovial fluids examined (one per subject) with subject characteristics. The three groups were divided into those with active and inactive joints. Median PPi and NTPase data are shown graphically in the figure. Table 2 provides an analysis of all the data. The principal findings were:

1 The order of PPi concentrations and NTPase activities was pyrophosphate arthropathy $>$ osteoarthritis $>$ rheumatoid arthritis, with highly significant differences between all groups $(\mathrm{p}<0.001)$.

2 In pyrophosphate arthropathy both $\mathrm{PPi}$ and NTPase were higher than in normal controls. In rheumatoid arthritis PPi concentrations were lower than in controls, though NTPase activities were the same. Results for osteoarthritis, however, were the same as those for normal controls.

3 The inflammatory state of the knee at the time of aspiration had little effect on synovial fluid findings in osteoarthritis. In pyrophosphate arthropathy, however, PPi concentrations were 
Table 1 Median (interquartile range) of inorganic pyrophosphate and NTPase by disease category and clinical activity

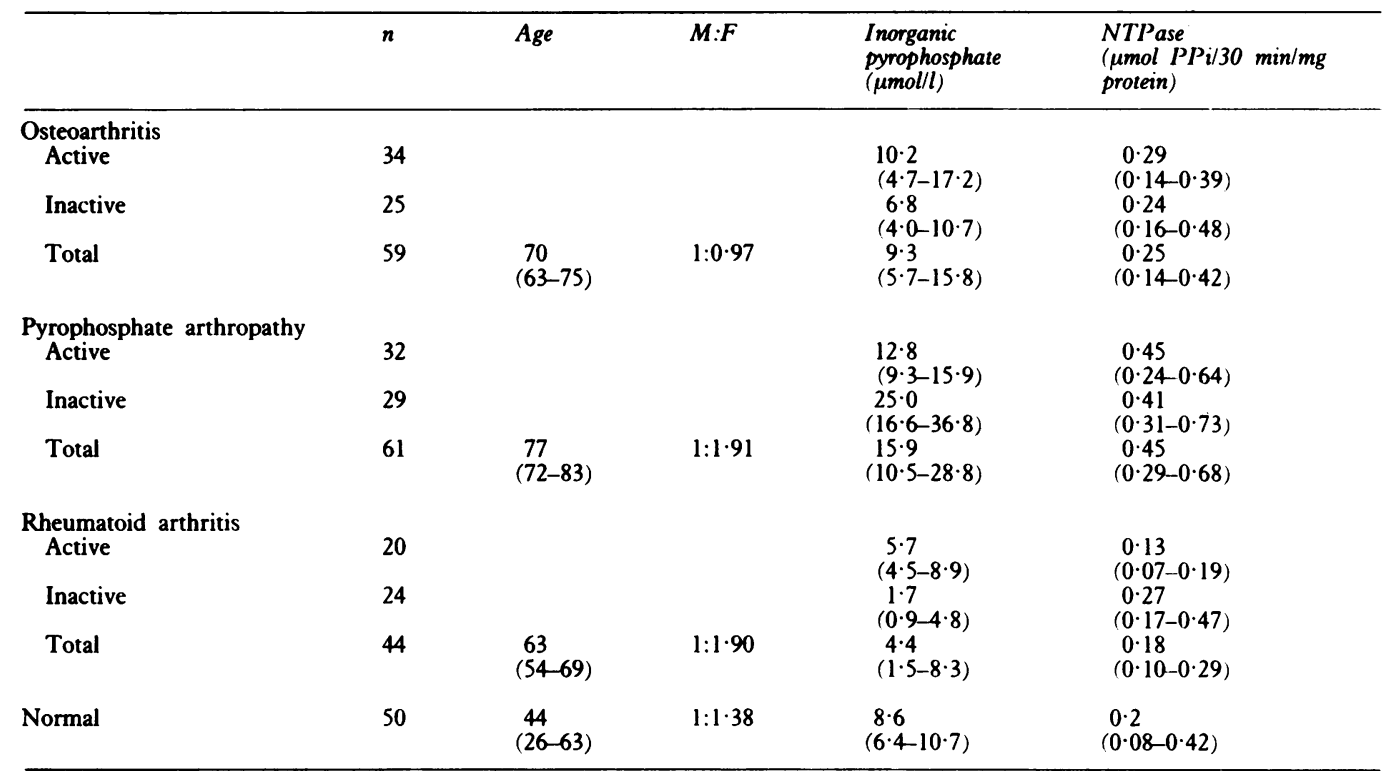

higher in inactive than in active knees, but there were no differences in NTPase. In rheumatoid arthritis, by contrast, PPi concentrations were higher in active joints, paradoxically accompanied by lower NTPase activity.

4 Neither PPi nor NTPase correlated with age in any group.

5 There was no correlation between the concentration of PPi and NTPase activity in any group.

\section{Discussion}

Several studies have examined PPi metabolism in patients with evidence of CPPD crystal deposition. Urine ${ }^{72}$ and subsequently
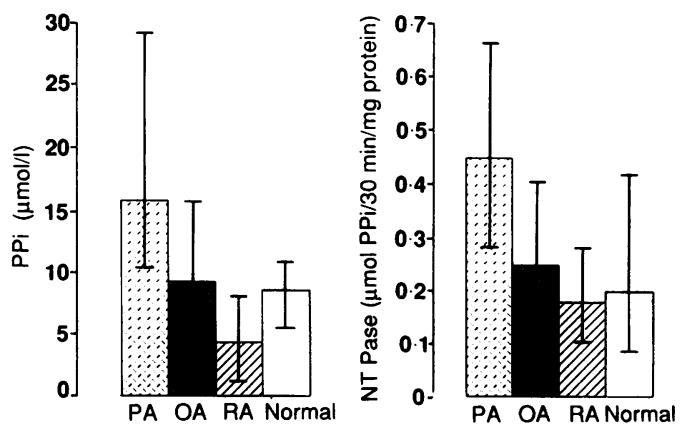

Median and interquartile range of inorganic pyrophosphate $(P P i)$ and nucleoside triphosphate pyrophosphatase (NTPase) activity by diagnostic group.

Table 2 Significant findings for intergroup and intragroup comparisons

\begin{tabular}{|c|c|}
\hline $\begin{array}{l}\text { Between group comparisons } \\
\text { PPi } \\
\begin{array}{l}\text { PA }>0 A=N o r m a l<R A \\
(p<0 \cdot 001) \quad(p<0 \cdot 001)\end{array} \\
\text { NTPase PA }>0 A, \text { Normal, RA (p } \\
\text { Normal }<R A(p\end{array}$ & $\begin{array}{l} \\
(0.001) \\
(0.002)\end{array}$ \\
\hline $\begin{array}{ll}\text { Within group comparisons } \\
\text { PPi } & \text { PA Inactive }>\text { PA Active } \\
& \text { RA Active }>\text { RA Inactive } \\
\text { NTPase } & \text { RA Inactive }>\text { RA Active }\end{array}$ & $\begin{array}{l}(\mathrm{p}<0.001) \\
(\mathrm{p}<0.001) \\
(\mathrm{p}<0.002)\end{array}$ \\
\hline
\end{tabular}

PPi=inorganic pyrophosphate; NTPase=nucleoside triphosphate pyrophosphatase; $\mathrm{PA}=$ pyrophosphate arthropathy; $\mathrm{OA}=$ osteoarthritis; RA=rheumatoid arthritis. plasma $^{35} 34$ concentrations were reported to be the same as for normal controls. With development of more sensitive assays PPi concentration was estimated in synovial fluid and found to be greater than in matched plasma, the increase being reported to correlate with radiographic deterioration. ${ }^{8}$ Smaller increases of synovial fluid PPi are also reported in osteoarthritis and in joints containing 'basic calcium phosphate' but not CPPD crystals ${ }^{35}$; the presence of consistently higher concentrations in synovial fluid than in matched plasma suggests local production within the joint. Synovial fluid NTPase activity has also been found to be increased when PPi is increased, suggesting that this enzyme is the major source of local PPi production. $^{1}$

This study therefore accords with previous reports in showing $(a)$ higher synovial fluid PPi concentrations and NTPase activities in pyrophosphate arthropathy than in osteoarthritis and $(b)$ lower values in rheumatoid arthritis than in pyrophosphate arthropathy or osteoarthritis. For example, Rachow et al, ${ }^{35}$ using the radiometric method of Cheung and Suhadolnik, ${ }^{19}$ found significant differences in $\mathrm{PPi}$ concentrations in synovial fluid from various joints (knees, shoulders, hips) of $\mathbf{4 0}$ patients with osteoarthritis and 27 with pyrophosphate arthropathy (mean (SD) 14 (5) and 18 (8) $\mu \mathrm{mol} / \mathrm{l}$ respectively); correspondingly higher NTPase activity was found in pyrophosphate arthropathy. Silcox and McCarty, using a differential colorimetric method, measured synovial fluid PPi in 35 patients with osteoarthritis, 29 with pyrophosphate arthropathy, and 12 with rheumatoid arthritis and found mean (range) concentrations of $9 \cdot 2$ (3-35), 9.8 $(4-24)$, and $4 \cdot 2(2-8) \mu \mathrm{mol} / 1$ respectively ${ }^{8}$; the differences between rheumatoid arthritis and both osteoarthritis and pyrophosphate arthropathy were significant (Student's $t$ test). Although these studies and ours agree about the differences between diagnostic categories, reported numerical values differ. This in part might reflect patient selection, differences in 
PPi assay technique, and for NTPase estimations differences in correction to total (present study) rather than specific ${ }^{26}$ proteins. Nevertheless, these differences are small and group values are within the same order of magnitude. Overall, such findings support the contention that local production of PPi relates to CPPD deposition, and that increased NTPase activity is a likely mechanism²; low values of $\mathrm{PPi}$ and NTPase in rheumatoid arthritis may also help to explain the negative association between rheumatoid arthritis and CPPD deposition. ${ }^{36}$

Certain observations, however, are not readily explained by NTPase regulated metabolism of PPi. In rheumatoid arthritis, for example, low concentrations of PPi occur in the presence of normal NTPase activity, and the lack of a direct correlation between PPi and NTPase in any group suggests that other factors may have a major effect on PPi concentrations. The importance of PPi metabolism in predisposition to sporadic CPPD crystal deposition must also be questioned. ${ }^{37} 38$ Various factors may inhibit or promote crystal nucleation and growth, ${ }^{37} 39$ and rates of crystal clearance and dissolution must be considered: the solubility product (calcium $\times \mathrm{PPi}$ ) may therefore not be the single most important determinant. The significance of factors apart from PPi is further emphasised by the discrepancy between the strong association between aging and CPPD deposition, and the apparent lack of age related change of PPi or NTPase in this study and in a larger cohort of normal subjects. ${ }^{39}$ Temporal fluctuation in PPi concentrations is a further factor which has been poorly considered: differing increases of PPi influence not only CPPD but also apatite crystal formation, ${ }^{40}$ and estimation at one timepoint may not be relevant to the crystals currently present. For this reason, cross-sectional studies relating single synovial fluid findings to 'radiographic damage' ${ }^{8}$ are difficult to interpret, prospective studies being required to determine such relationships more precisely.

We recognise that there are inherent problems with all studies estimating concentrations in synovial fluid due to undetermined joint volumes and synovial mass, the unknown dynamics of production, breakdown and clearance, and the question of correction to other synovial fluid constituent concentrations. Furthermore, throughout such experiments it is generally assumed that synovial fluid concentrations reflect those in cartilage and we accept that this may not be valid.

In the study by Silcox and McCarty paradoxically lower concentrations of $\mathrm{PPi}$ in acute pseudogout than in chronic pyrophosphate arthropathy $(\mathrm{n}=18$; mean $5.4 v 10.4 \mu \mathrm{mol} / \mathrm{l})$ suggested an association between inflammation and PPi concentrations. ${ }^{8}$ This was attributed to increased synovial flow and faster clearance of PPi. To date, however, few synovial fluid studies have characterised joints according to inflammatory state. There is no generally agreed method of clinically assessing knee inflammation, but the system we chose uses a summated score of six features and has been
Table 3 Factors influencing synovial fluid component concentration

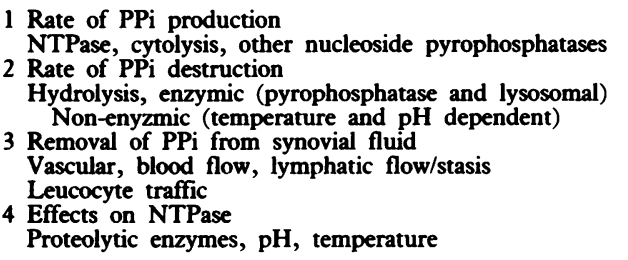

Proteolytic enzymes, $\mathrm{pH}$, temperature

$\mathrm{PPi}=$ inorganic pyrophosphate $\mathrm{NTPase}=$ nucleoside triphosphate pyrophosphatase.

shown to correlate with complement activation in several arthritides. ${ }^{28}$ Interestingly, we found the presence of such inflammation to have different associations in each condition: in rheumatoid arthritis it associated with increased PPi but lowered NTPase; in pyrophosphate arthropathy it associated with reduced $\mathrm{PPi}$; and in osteoarthritis it had no discernible effect. Although such findings are difficult to explain, there are a number of ways in which inflammation might be expected to influence $\mathrm{PPi}$ metabolism (table 3) and the relative effects of these may vary in different conditions. The apparent lack of association with inflammation in osteoarthritis is of special interest: unlike rheumatoid arthritis, synovial fluid parameters, such as complement activation products ${ }^{28}$ or $\mathrm{PPi}$, were insensitive and did not reflect even florid clinical inflammation in osteoarthritis, suggesting that the mechanisms of inflammation in rheumatoid arthritis and osteoarthritis are different. Such discrepancies indicate that comparative synovial fluid studies should consider degree of inflammation as well as diagnostic categories.

Data on non-cadaveric, normal, human synovial fluids are understandably sparse. For PPi the results for only five normal joints have been published, ${ }^{5}$ giving a mean value of $3.6 \mu \mathrm{mol} / 1$; we are unaware of any data for NTPase. In this study, using a different assay, we found the mean PPi concentration from 50 normal knees to be $9.3 \mu \mathrm{mol} / \mathrm{l}$; values for normal synovial fluid NTPase activity are also presented. Increases of synovial fluid PPi and NTPase in pyrophosphate arthropathy, compared with normal or osteoarthritis, may reflect increased biosynthetic activity within the joint rather than implying any specific alteration in PPi metabolism. Such an interpretation would accord with the 'hypertrophic response' (florid osteophytosis, cysts, remodelling) and possible good outcome ${ }^{3}$ which have been associated with pyrophosphate arthropathy. Conversely, low synovial fluid concentrations of PPi in rheumatoid arthritis may reflect enzymic hydrolysis of PPi to orthophosphate, or an 'atrophic' articular response to insult.

We would like to thank the Arthritis and Rheumatism Council for financial support, and Professor R G G Russell and Dr Alison $M$ Caswell for help in establishing the assays for PPi and NTPase.

1 Russell R G G. Metabolism of inorganic pyrophosphate (PPi). Arthritis Rheum 1976; 19: 465-78.

2 Doherty M, Dieppe P. Crystal deposition disease in the elderly. Clin Rheum Dis 1986; 12: 97-116. 
3 Doherty M. Pyrophosphate arthropathy -a clinical study. Cambridge: Cambridge University, 1988. (MD thesis.)

4 Silcox D C, McCarty D J. Elevated inorganic pyrophosphate concentrations in synovial fluids in osteoart

5 Altman R D, Muniz O E, Pita J C, Howell D S. Articular chondrocalcinosis. Microanalysis of pyrophosphate (PPi) in chondrocalcinosis. Microanalysis of pyrophosphate (PPi) in synovial fluid plasma. Arthritis Rheum 1973; 16: 171-8. McCarty D J, Solomon S D, Warnock M L, Paloyan E. Inorganic pyrophosphate concentrations in the synovia fluid of arthritic patients. F Lab Clin Med 1971; 78: 216-29.

7 Russell R G G, Bisaz S, Fleisch H. Inorganic pyrophosphate in plasma, urine and synovial fluid of patients with pyrophosphate arthropathy (chondrocalcinosis or pseudogout). Lancet 1970; ii: 899-902

8 Silcox D C, McCarty D J. Measurement of inorganic pyrophosphate in biological fluids. Elevated levels in some patients with osteoarthritis, pseudogout, acromegaly, and uremia. F Clin Invest 1973; 52: 1863-70.

9 Good A E, Starkweather W H. Synovial fluid pyrophosphate (PPPH) in pseudogout. Arthritis Rheum 1969; 12: 298 .

10 Rachow J W, Ryan L M. Partial characterization of synovial fluid nucleotide pyrophosphohydrolase. Arthritis Rheum 1985; 28: 1377-83.

11 Jacobelli S, Kettlun A M, Sapag-Hagar M. Inorganic pyrophosphatase activity of the synovial fluid. Arthritis Rheum 1978; 21: 447-52.

12 Micheli A, Po J, Fallet G H. Measurement of soluble pyrophosphate in plasma and synovial fluid of patients with various rheumatic diseases. Scand $\mathcal{f}$ Rheumatol 1981; 10: $237-40$

13 Ryan L M, Wortmann R L, Karas B, McCarty D J. Cartilage nucloeside triphosphate (NTP) pyrophosphohydrolase. I. Identification as an ecto-enzyme. Arthritis Rheum 1984; 27: 404-9.

14 Ryan L M, Wortmann R L, Karas B, McCarty D J. Cartilage nucleoside triphosphate pyrophosphohydrolase. II. Role in extracellular pyrophosphate generation and nucleotide extracellular pyrophosphate generation and

15 Rachow J W, Ryan L M. Adenosine triphosphate pyrophosphohydrolase and neutral inorganic pyrophosphatase in pathologic joint fluids. Elevated pyrophosphohydrolase in calcium pyrophosphate dihydrate crystal deposition disease. Arthritis Rheum 1985; 28: 1283-8.

16 McGuire M B, Colman C H, Baghat N, Russell R G G. Radiometric measurement of pyrophosphate in cell cultures. Biochem Soc Trans 1980; 8: 529-30.

17 Lust G, Seegmiller J E. A rapid, enzymatic assay for measurement of inorganic pyrophosphate in biological samples. Clin Chem Acta 1976; 66: 241-9.

18 Cartier P H, Thuillier L. Measurement of inorganic pyrophosphate in biological fluids and bone tissues. Anal phosphate in biological flui

19 Cheung C P, Suhadolnik R J. Analysis of inorganic pyrophosphate at the picomole level. Anal Biochem 1977; 83: 61-3.

20 Flodgaard H. Isotope derivative method for determination of microquantities of inorganic pyrophosphate in biological material. Eur F Biochem 1970; 15: 273-9.

21 Johnson J C, Shanoff M, Bass S T, Boezi J A, Hansen R G. An enzymic method of determination of inorganic pyrophosphate and its use as an assay for RNA polymerase. Anal Biochem 1968; 26: 137-45.

22 Caswell A M, Russell R G G. Identification of ecto-nucleoside triphosphate pyrophosphatase in human articular chondrocytes in monolayer culture. Biochim Biophys Acta 1985; 847: $40-7$.

23 Howell D S, Muniz O, Pita J C, Enis J E. Extrusion of pyrophosphate into extracellular media by osteoarthritic cartilage incubates. $\mathcal{J}$ Clin Invest 1975; 56: 1473-80.

24 Tenenbaum J, Muniz O, Schumacher H R, Good A E Howell D S. Comparison of phosphohydrolase activities from articular cartilage in calcium pyrophosphate deposition disease and primary osteoarthritis. Arthritis Rheum 1981 24: 492-500.

25 Ryan L M, Cheung H S, McCarty D J. Release of pyrophosphate by normal mammalian articular hyaline and fibrocartilage in organ culture. Arthritis Rherm 1981; 24: 1522-7.

26 Muniz O, Pelletier J-P, Martel-Pelletier J, Morales S, Howell D S. NTP pyrophosphohydrolase in human chondrocalcinotic and osteoarthritic cartilage. I. Some biochemica characteristics. Arthritis Rheum 1984; 27: 186-92.

27 Howell D S, Martel-Pelletier J, Pelletier J-P, Morales S, Muniz O. NTP pyrophosphohydrolase in human chondrocalcinotic and osteoarthritic cartilage. II. Further studies on histologic and subcellular distribution. Arthritis Rheum 1984; 27: 193-9.

28 Doherty M, Richards N, Hornby J, Powell I. Relationship between synovial fluid $\mathrm{C} 3$ degradation products and local joint inflammation in rheumatoid arthritis, osteoarthritis and crystal associated arthropathy. Ann Rheum Dis 1988; 47: $190-7$.

29 Kornberg A. On the metabolic significance of phosphorolytic and pyrophosphorolytic reactions. In: Kasha M, Pullman B, eds. Horizons in biochemistry. York: Academic Press, 1962: 251-64. (Albert Szent-Gyorgyi dedicatory volume.)

$30 \mathrm{Khym}$ J X. An analytical system for rapid separation of tissue nucleotides at low pressures on conventional anion exchangers. Clin Chem 1975; 21: 1245-52.

31 Sugino Y, Miyoshi Y. The specific precipitation of orthophosphate and some

32 Pflug M, McCarty phate excretion in pseudogout. Arthritis Rheum 1969; 12: 228-31.

33 Ryan L M, Kozin F, McCarty D J. Quantification of human plasma inorganic pyrophosphate. II. Biologic variables. plasma inorganic pyrophosphate.

34 Ryan L M, Kozin F, McCarty D J. Quantification of human plasma inorganic pyrophosphate. I. Normal values in osteoarthritis and calcium pyrophosphate dihydrate crystal deposition disease. Arthritis Rheum 1979; 22: 886-91.

35 Rachow J W, Ryan L M, McCarty D J, Halverson P C Synovial fluid inorganic pyrophosphate concentration and nucleotide pyrophosphohydrolase activity in basic calcium phosphate deposition arthropathy and Milwaukee shoulder phosphate deposition arthropathy and Milwauke

36 Doherty M, Dieppe P A, Watt I. Low incidence of calcium pyrophosphate dihydrate crystal deposition in rheumatoid arthritis with modification of radiographic features in coexistent disease. Arthritis Rheum 1984; 27: 1002-9.

37 Ohira T, Ishikawa K, Masuda I, et al. Histologic localisation of lipid in the articular tissues in calcium pyrophosphate dihydrate deposition disease. Arthritis Rherom 1988; 31 : $1057-62$.

38 Doherty $\mathbf{M}$, Pattrick M, Hamilton E. Inorganic pyrophosphate, nucleoside triphosphate pyrophosphatase and cartilage fragments in normal synovial fluid. Arthritis Rheum 1988; 32 (suppl): S69.

39 Cheng P, Pritzker K P H. Inhibition of calcium pyrophosphate dihydrate crystal formation: effects of carboxylate ions. Calcif Tissue Int 1988; 42: 46-52.

40 Chuck A J, Pattrick M G, Hamilton E, Wilson R, Doherty $M$. Crystal deposition in hypophosphatasia: a reappraisal. Ann Rheum Dis 1989; 48: 571-6. 